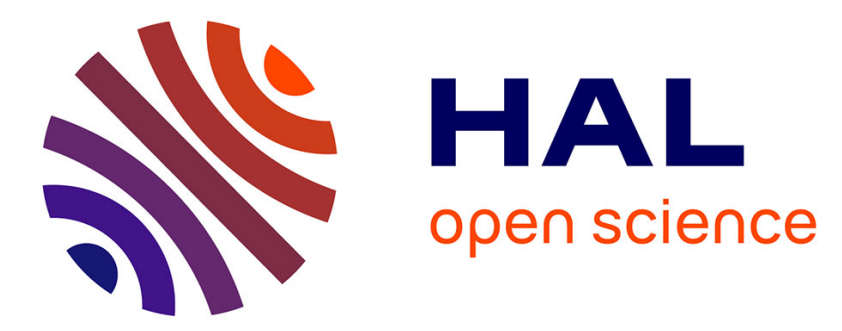

\title{
3D manufacturing tolerancing with probing of a local work coordinate system
}

Marie Royer, Bernard Anselmetti

\section{To cite this version:}

Marie Royer, Bernard Anselmetti. 3D manufacturing tolerancing with probing of a local work coordinate system. International Journal of Advanced Manufacturing Technology, 2016, 84 (9), pp.21512165. 10.1007/s00170-015-7797-4 . hal-01326951

\section{HAL Id: hal-01326951 https://hal.science/hal-01326951}

Submitted on 6 Jun 2016

HAL is a multi-disciplinary open access archive for the deposit and dissemination of scientific research documents, whether they are published or not. The documents may come from teaching and research institutions in France or abroad, or from public or private research centers.
L'archive ouverte pluridisciplinaire HAL, est destinée au dépôt et à la diffusion de documents scientifiques de niveau recherche, publiés ou non, émanant des établissements d'enseignement et de recherche français ou étrangers, des laboratoires publics ou privés.

\section{(ㅇ)(1) $\$$}

Distributed under a Creative Commons Attribution - NonCommercial - NoDerivatives| 4.0 


\title{
3D manufacturing tolerancing with probing of a local work coordinate system
}

\author{
Marie Royer (a,b) \\ Bernard Anselmetti (a)
}

a. LURPA, ENS Cachan, Univ. Paris-Sud, Université Paris-Saclay, 94235 Cachan, France

b. SNECMA Evry-Corbeil, Rue Henri Auguste Desbruères, 91003 Evry, France

\author{
The International Journal of Advanced Manufacturing Technology \\ June 2016, Volume 84, Issue 9, pp 2151-2165
}

\begin{abstract}
The safety and performance requirements for mechanisms are such that the necessary accuracy of part geometry is difficult to reach using classical manufacturing processes. This paper proposes a manufacturing tolerance stack-up method based on the analysis line method. This technique enables both the analysis and the synthesis of ISO manufacturing specifications through a new approach which relies on production specifications, adjustment specifications and their analysis to stack up the 3D resultant. The originality of the method resides in the $3 \mathrm{D}$ calculation for location requirements, which takes into account angular effects and probing operations on numerical-control machine-tools in order to define a local Work Coordinate System (WCS). For achieving tolerance analysis, deviations are modelled using Small-Displacement Torsor. This tolerance analysis method enables one to determine explicit three-dimensional linear relations between manufacturing tolerances and functional requirements. These relations can be used as constraints for tolerance optimization.
\end{abstract}

Keywords: Manufacturing ISO tolerancing Tolerance analysis Specification synthesis Analysis line method Probing 


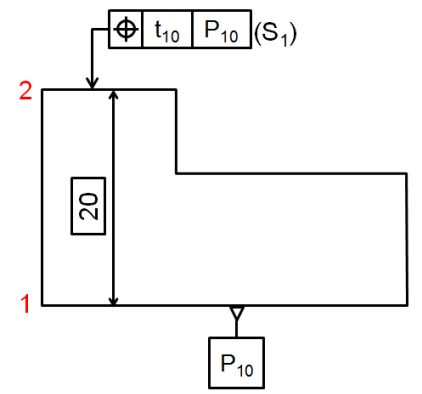

(a) Production specification in phase 10

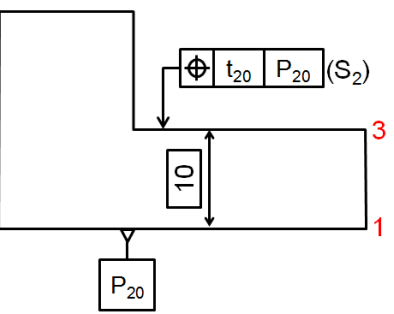

(b) Production specification in phase 20

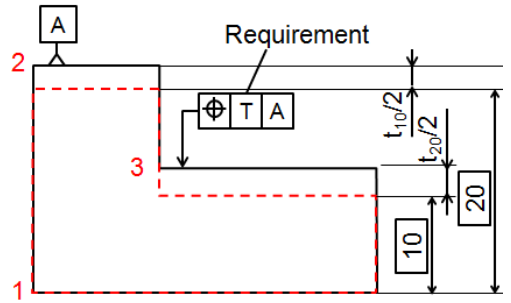

(c) Functional requirement and $1 \mathrm{D}$ transfer

Figure 1: 1D manufacturing transfer

\section{Definition of the problem}

\subsection{Manufacturing tolerancing}

\subsubsection{The context}

This paper falls within the industrial framework of a manufacturing company which produces precision machined parts in small-volume production runs, for example in the aeronautical industry. The Computer Aided Design (CAD) model describes the finished part along with the functional requirements. First a process plan is established by specifying all the stage models and set-ups. The manufacturing engineer must define the manufacturing specifications for each manufacturing phase.

These specifications must be controlled by the mill machinist. The manufacturing specifications are expressed according to ISO standards $[11,12]$ and must be defined between active surfaces of the phase. The active surfaces of a manufacturing phase consist in the surfaces which are manufactured in that phase plus the surfaces of the datum system of the phase (the surfaces of the workpiece in contact with the part holder and the surfaces identified through probing).

\subsubsection{Principle of manufacturing tolerances analysis}

Fig. 1 describes the principle of manufacturing transfer for an elementary process plan consisting of two phases. The calculation is carried out in $1 \mathrm{D}$, which means that angular effects are disregarded. The requirement considered is the location of surface 3 with a tolerance $\mathrm{T}$ with respect to datum A defined on surface 2 (Figure 1c). Surfaces 2 and 3 are manufactured in two separate phases. The two production specifications $S_{1}$ and $S_{2}$ are shown in Figures 1a and 1b. The calculation model of the minimum or maximum resultant assumes a $\pm \frac{t}{2}$ translation of the machined surfaces with respect to their nominal positions. For the requirement being studied, the condition to be satisfied is:

$$
t_{10}+t_{20} \leq T
$$

Figure 2c shows the influence of angular defects and the necessity of a 3D calculation. The distance between point $\mathrm{F}$ and datum $\mathrm{A}$ shows that an additional term $d=t_{10} \frac{L}{E}$ must be introduced. Accounting for this effect leads to the following relation: 


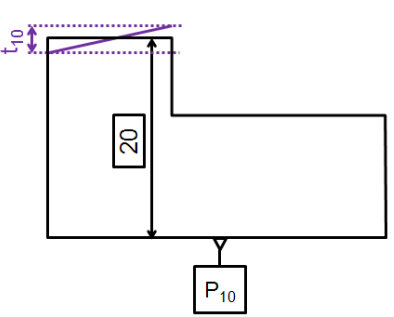

(a) The worst case in phase 10

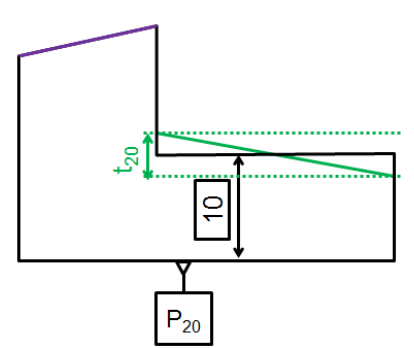

(b) The worst case in phase 20

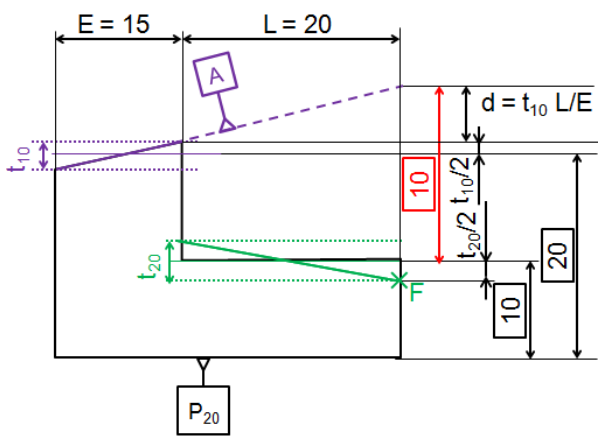

(c) 3D transfer

Figure 2: Accounting for 3D effects in the transfer

$$
t_{10}+t_{20}+2 \frac{L}{E} t_{10} \leq T
$$

with $L=20$ and $E=15$.

In a context in which the allowable defects in the finished part are very small compared to the large dimensions of the parts, these three-dimensional effects must be taken into account in the tolerance analysis. In order to limit the angular effects $(d)$, one must control the maximum slope by adding an orientation specification in phase 10 .

\subsection{Probing on Numerical-Control (NC) machine-tools}

In the context of the production of parts on $\mathrm{NC}$ machine-tools, the number of machining operations to be carried out in one phase can be very large. Indeed, reducing the number of phases enables one to improve the quality of the finished parts by reducing the deviations caused by the handling of the workpiece.

The latest probing technologies on NC machine-tools enable one to define a local Work Coordinate System (WCS) in order to machine surfaces directly in relation to preexisting surfaces of the part, and thus to limit transfers and avoid overly strict manufacturing tolerances. Figure 3 shows an actual case taken from the aeronautical industry. This (extremely simplified) engine part is manufactured on a vertical lathe. In this phase, surface 6 of the part is set-up on the jaws. WCS $R_{30.1}$ of the part is defined on that surface in order to machine a series of surfaces, including groove 5 at a distance of $50 \mathrm{~mm}$. Surface 3 was manufactured in a previous phase. In order to comply with a stringent functional requirement between surfaces 2 and 3, the position of surface 3 is measured in the part's coordinate system using a probe, thus defining a local WCS $R_{30.2}$ by shifting the origin with respect to WCS $R_{30.1}$. Then surface 2 is precision machined in this local WCS $R_{30.2}$.

\subsection{State of the art}

The only methodologies that are available to most companies in order to determine manufacturing specifications are highly empirical and most often manual because there is no commercial software tool. This problem is becoming increasingly difficult for 4 reasons:

- Companies have adopted ISO 3D tolerancing 


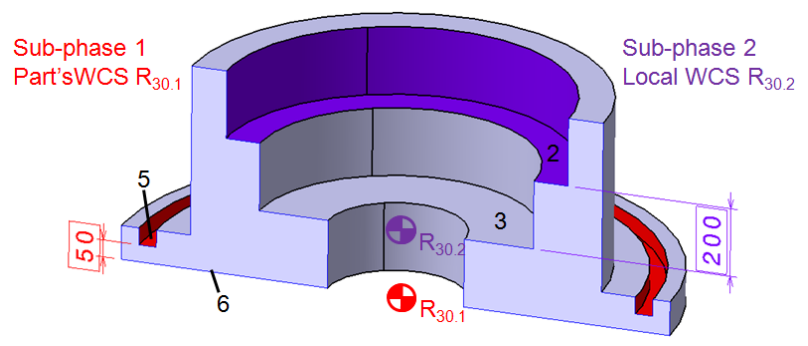

Figure 3: Machining operations in a part's WCS and in a local WCS

- Companies are seeking product/process/metrology numerical continuity

- Tolerance optimization is mandatory for both economic and performance reasons

- Production tools are becoming more and more complex (5-axis milling machines, onmachine measurement...)

For all these reasons, manual approaches have become insufficient. In the functional tolerancing approach, Salomons [17] identified four activities, whose definitions can be extended to manufacturing tolerancing:

- The first activity, called tolerance type specification by Chase and Salomons [8, 16] or specification synthesis by Anselmetti [1], is the determination of the types of manufacturing specifications which are necessary in order to control the functional requirements. This is a qualitative approach.

- The second activity, called tolerance value specification by Chase and Salomons [8, 16], is the choice of the tolerance values based on the company's experience.

- The third activity is, according to the definition proposed by Salomons [15], tolerance analysis, whose objective is to verify that the requirements are satisfied for the tolerance values given. The maximum or minimum value of the resultant can be determined by numerical simulation. One can also express the resultant as a weighted sum of the manufacturing tolerances:

$$
\sum_{i=0}^{n} k_{i} t_{i} \leq T
$$

where $t_{i}$ stands for the $n$ manufacturing tolerances and $T$ is the tolerance on the functional requirement set by the engineering department.

- If all the functional requirements are described by conditions of the form (3), the fourth step, called tolerance synthesis enables one to optimize the allocation of the manufacturing tolerances. A comparative study of several tolerance synthesis methods was presented by Kusiak [13].

Two families of approaches to tolerance analysis can be distinguished.

The first approach consists in performing operations over domains which represent the deviations of the surfaces from the nominal geometry, which enables one to simulate the consequences of dispersions related to the manufacturing process on the geometry of the finished part. Villeneuve and Vignat $[18,19,20]$ described the Model of Manufactured Parts (MMP), which represents the actual geometry of the part. To develop the MMP, the deviations induced by 


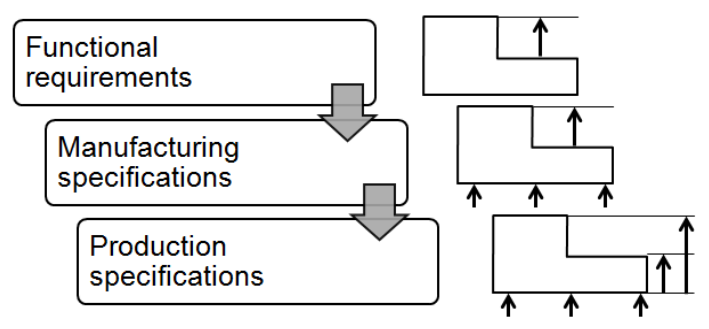

(a) The current process

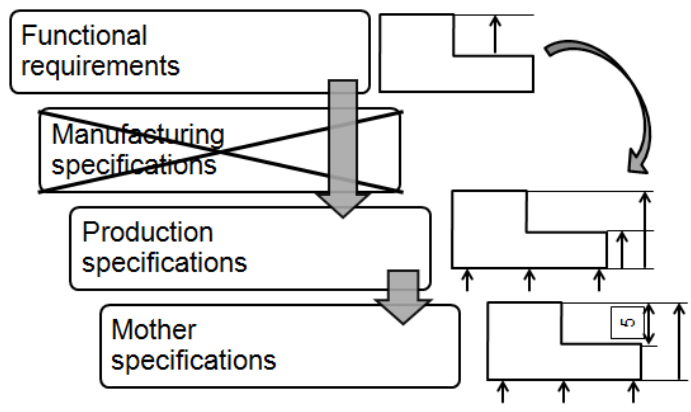

(b) The new approach

Figure 4: The current process and the new approach

machining and positioning dispersions are expressed as Small-Displacement Torsors [7]. The parameters which define these torsors are constrained by dependency relations. Then, two types of simulations are proposed in order to generate the MMP:

- the search for the worst case by means of an optimization calculation,

- the generation of a statistical MMP set by Monte-Carlo simulation.

More recently, Haghighi defined M-Maps, i.e. domains which model the stacking-up of manufacturing defects [10]. M-Maps, which are obtained by performing Minkowsky sums over domains of variation, are derived from Tolerance Maps (T-Maps), which are dedicated to the analysis of functional tolerances in order to verify that a functional requirement on an assembly is met $[9]$.

The second family of approaches consists in propagating the deviations associated with each manufacturing phase to the rest of the process by means of a calculation. This is the type of approach which is often used in industry with the $\Delta l$ method. This method developed by Bourdet [6] offers the possibility to translate functional and manufacturing requirements into sets of manufacturing dimensions through a one-dimensional calculation. This method was extended to two-dimensional calculations of turning operations by Anselmetti [2]. In addition, three-dimensional calculation methods based on the Small-Displacement Torsor principle were proposed by Ballot and Laifa [5, 14]. The defects associated with each phase are modeled as the 6 components of a torsor. The resultant torsor is obtained by summing up the components of these torsors, then applied to the various points of the surface specified by the requirement. With the analysis line method, which was initially dedicated to functional tolerancing [3], Anselmetti applies the sum of the influences of each phase directly onto the points of the surface specified by the requirement [4]. 


\subsection{The proposed method}

The first contribution of this article is a specification synthesis and tolerance analysis method using a three-dimensional approach based on the analysis line method [2, 4]. Small-Displacement Torsors are used to model the defects allowed by the manufacturing tolerance zones, regardless of their cause (setting-up defects, tool-related defects, part deformation...). The resulting transfer relations are linear inequations in terms of the manufacturing tolerances, which, thus, lend themselves easily to the synthesis of the tolerances using an optimization algorithm [3]. In this transfer method, the manufacturing datum systems must be defined as datum targets which describe either the setting-up of the part or the measuring points used to define the programming coordinate system for the machining operations. Then, the manufacturing specifications expressed in this datum system can be controlled directly by the mill machinist.

The second contribution of this paper is a new approach to the choice of suitable manufacturing specifications for NC machining which enable an independent adjustment of each tool.

The third contribution of this paper is the integration into this method of the specificity of the construction of the programming coordinate system through probing on $\mathrm{NC}$ machines.

\section{Production specification synthesis and mother speci- fications}

\subsection{The classical approach}

Figure 4a illustrates the current manufacturing tolerancing process. Starting from the functional requirements, a set of necessary and sufficient manufacturing specifications to guarantee that these functional requirements are met is determined. Each manufacturing specification associates a set of active surfaces in a phase. Then, in order to enable each tool to be adjusted independently, this type of manufacturing specification on surface manufactured by many tools is divided into production specifications which set up each machined surface directly with respect to the datum system of the phase.

\subsection{The new approach}

In Figure 4b, a new approach is proposed. Starting from the functional requirements, the production specifications are determined between machined surface and the datum system. Then, these production specifications enable the analysis and the synthesis of the tolerances. These tolerances help the manufacturing engineer choose the detailed machining protocol (rough, semi-finish) and the various tools. At this point, all the surfaces machined by the same tool are assumed to be perfectly located and oriented with respect to one another, except for a small random dispersion. Thus, it suffices to control the most stringent production specification to qualify the tool's adjustment. At Snecma, the chosen specification is called the mother specification. The other specifications which depend on that tool are called the daughter specifications. Thus, these are manufactured at a stricter tolerance than necessary, which enables one to carry out a new distribution of the tolerances on the mother specifications.

This new approach eliminates a major difficulty with the classical method, which is the expression in terms of ISO standards of some manufacturing specifications involving several surfaces machined in the same phase. 


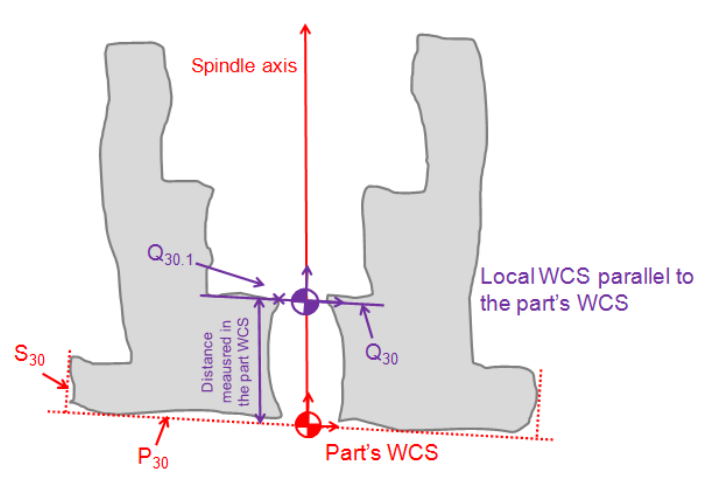

Figure 5: Construction of the local programming WCS

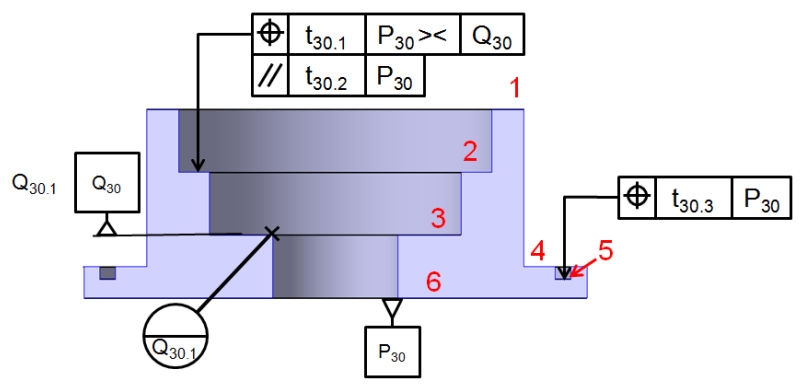

Figure 6: Production specifications with a local WCS

\section{The local programming Work Coordinate System (WCS)}

\subsection{Definition of a local WCS on a NC machine-tool}

The general principle of machining using an NC machine-tool consists in defining a part's WCS on the part's setting-up surfaces in the part holder, then machining several surfaces in that WCS.

One can also define a local programming WCS on some surfaces of the part which are manufactured in this phase or were manufactured in a previous phase. In order to do that, a touch-trigger probe is mounted in the spindle to measure the positions of datum points with respect to the part's initial coordinate system. The local WCS is constructed by shifting the origin.

In 3-axis milling, WCS shifting can be carried out only in translation. Thus, the new WCS is defined to be parallel to the original one and does not coincide exactly with the probed surface. The residual angular deviation must be taken into account in the manufacturing transfer.

\subsection{Manufacturing specifications in a local WCS}

For the phase presented in Figure 3, which is carried out by vertical turning, the part's WCS is defined (according to the direction of the machine-tool spindle) based on a point defined on primary surface $P_{30}$ and centered on cylinder $S_{30}$ (Figure 5). The local programming WCS is shifted along the Z-axis in order to go through measuring point $Q_{30.1}$.

Thus, there are two groups of active surfaces in this phase. Figure 6 presents the production specifications expressed according to ISO standards. The part's coordinate system is characterized by the part's datum plane $P_{30}$. For example, surface 5 is located with respect to this datum $P_{30}$. 


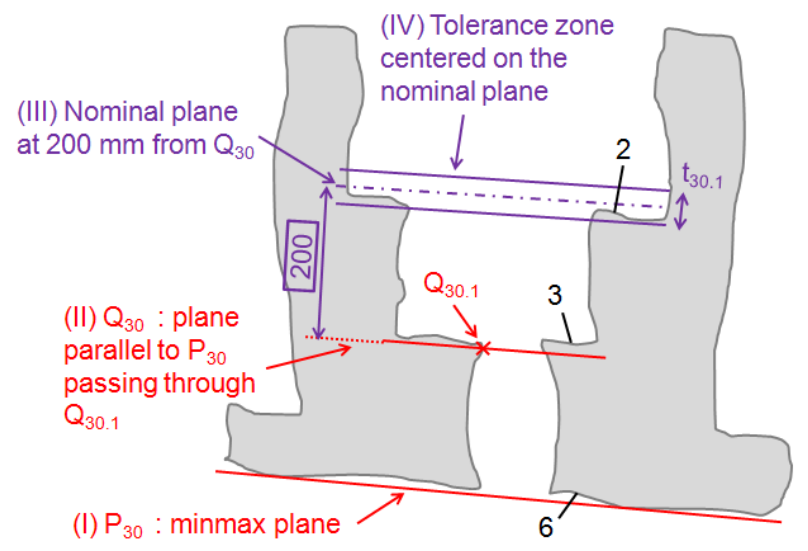

Figure 7: The construction of datum system $P_{30}><\mid Q_{30}$

The construction of this datum system, given in Figure 7, is carried out according to the following procedure:

- (I): Datum plane $P_{30}$, associated with real surface 6 (assumed to be a plane) $P_{30 \text {,real }}$, is constructed as tangent outside material minimizing the maximum deviation from $P_{30 \text {,real }}$ (Chebyshev's outside material criterion).

- (II): The datum system which corresponds to the local WCS defined in Figure 5 is written as

$P_{30}><\mid Q_{30}$. The modifier $><$ proposed by ISO standard [12] means that $P_{30}$ constrains the tolerance zone of the considered specification "only in orientation". Datum plane $Q_{30}$ is defined parallel to $P_{30}$ and tangent outside material to the set of the points probed on surface 3. Since there is only one probed point $Q_{30.1}, Q_{30}$ goes through that point.

- (III): Nominal plane 2 is $200 \mathrm{~mm}$ from $Q_{30}$.

- (IV): The tolerance zone, limited by two parallel planes $t_{30.1}$ apart, is centered on the nominal plane. The specification meets the requirement if the toleranced surface is included in the tolerance zone.

The ISO definition proposed here matches the construction of the local WCS in the machine. Since the manufacturing datum system is constructed parallel to datum $P_{30}$ and goes through the probed point $Q_{30.1}$, its orientation and its position depend on the orientation defect of surface $P_{30}$ and on the location defect of point $Q_{30.1}$.

\section{Manufacturing transfer using analysis lines}

\subsection{Industrial application}

\subsubsection{Definition drawing}

Figure 8 shows the simplified definition drawing of the part, which is a component of a large engine. The functional requirement being studied is a location of plane 2 with respect to datum A on plane 1. Figure 8 defines the surfaces and gives the main nominal dimensions of the part. 


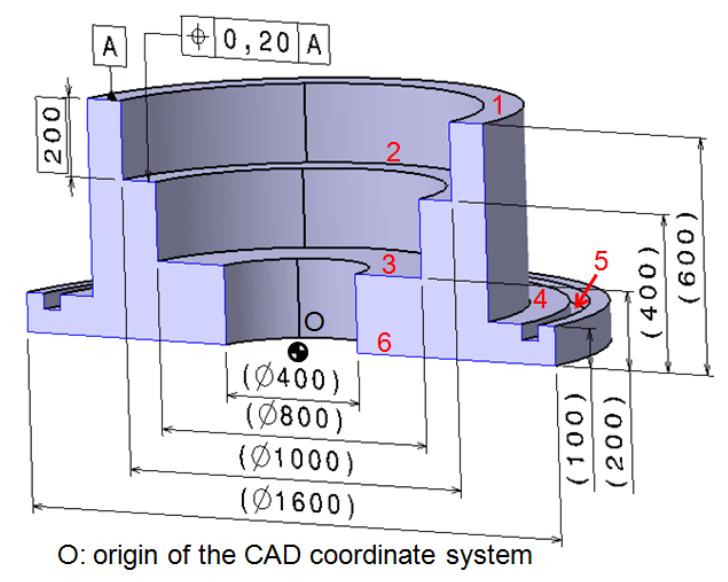

Figure 8: Definition drawing of the part

\subsubsection{The process plan}

Figure 9 presents the simplified process plan of the part being studied.

The blank (Figure 9a) is forged, then machined. In phase 10 (Figure 9b), the workpiece is set up on plane 4 in order to machine faces 1 and 6 . The production specifications will be expressed as positions or orientations with respect to datum $P_{10}$ associated with set-up surface 4 .

In phase 20 (Figure 9c), the workpiece is set up on plane 1 to machine 3 . The production specifications will be expressed as positions or orientations with respect to datum $P_{20}$ associated with set-up surface 1.

In phase 30 (Figure 9d), the workpiece is set up on plane 6 to machine 5 . The production specifications will be expressed as positions or orientations with respect to datum $P_{30}$ associated with set-up surface 6 . Then, in the same phase, a probe is used to measure the position of a point $Q_{30.1}$ on surface 3. A new local WCS $R_{30.2}$ is defined at this point (Figure 5). Surface 2 is machined in this WCS. The production specifications will be expressed as positions or orientations with respect to datum system $P_{30}><\mid Q_{30}$ associated with set-up surfaces 6 and 3 (Figure 6).

A study of the process plan and isostatism suffices to define the datum systems of each phase. The problem now consists in choosing the specifications and the tolerance values which guarantee that the requirement of Figure 8 is met. Angular effects are significant and require a three-dimensional transfer which is to be addressed by the analysis line method.

\subsection{The $R$ coordinate system on the datum system of the first phase}

In this method, the nominal model of the part includes the finished surfaces, the blank surfaces and the rough surfaces. This model is set up in a coordinate system $\mathrm{R}$ defined on the datum system of the first machining phase.

In the analysis line method, all geometric deviations are considered to be displacements of surfaces or points with respect to the corresponding nominal surfaces in system $\mathrm{R}$. These displacements are described by a torsor expressed at point $\mathrm{O}$ (the origin of the CAD model).

\subsection{Analysis of the requirement}

\subsubsection{The condition to be met}

The functional requirement which is transfered is the location of surface 2 with respect to datum $\mathrm{A}$ as described in Figure 8. 


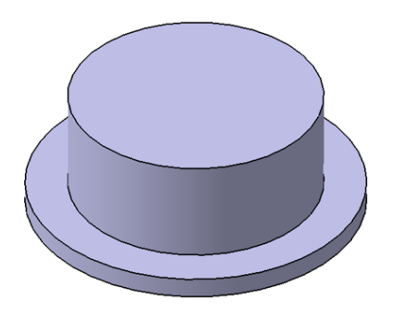

(a) Blank

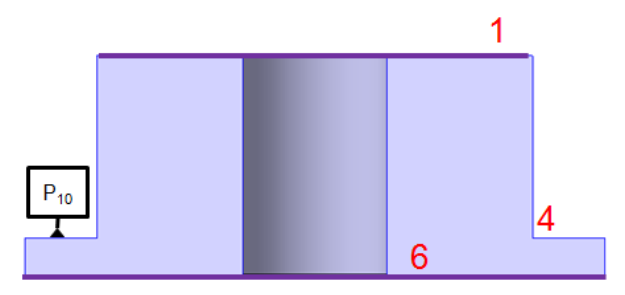

(b) Phase 10. Machined surfaces: 1,6

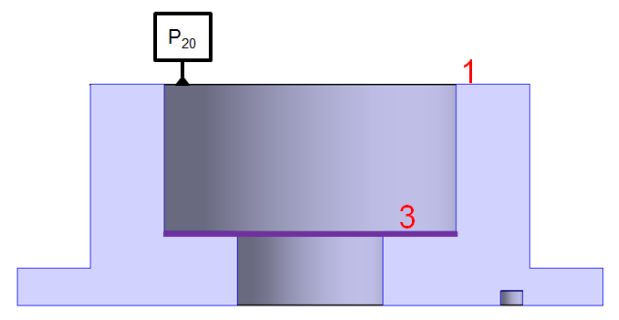

(c) Phase 20. Machined surface: 3

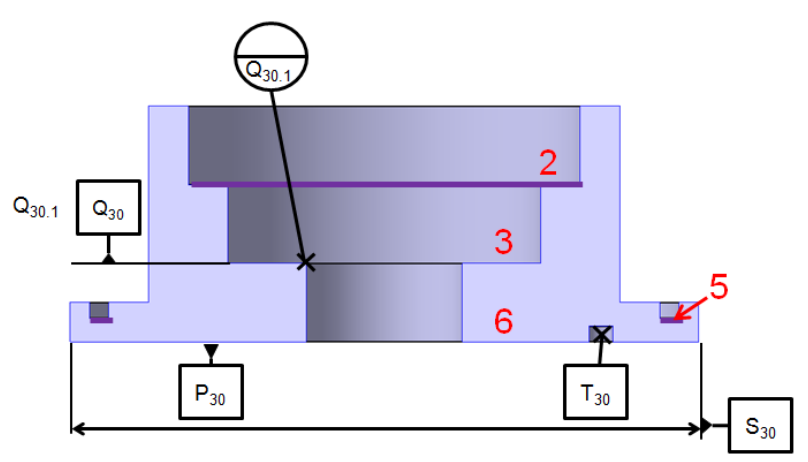

(d) Phase 30. Machined surfaces: 2, 5

Figure 9: Process plan 


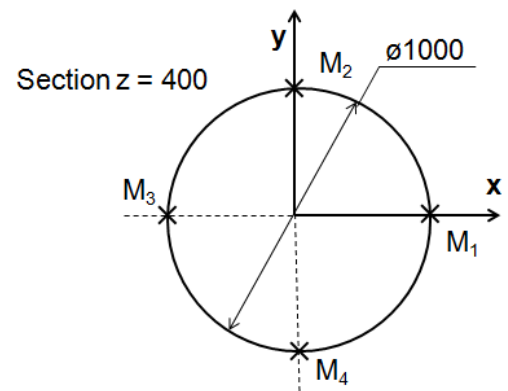

(a) Discretization of the toleranced surface 2

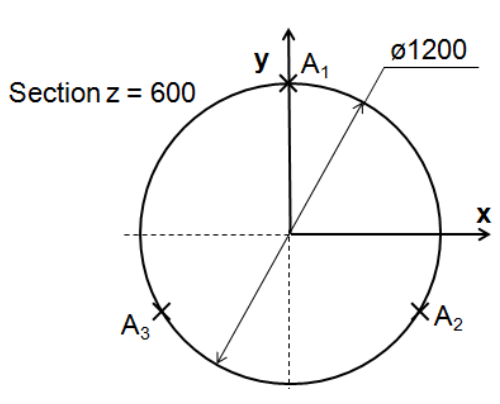

(b) Discretization of the datum surface 1

Figure 10: Discretization of the surfaces

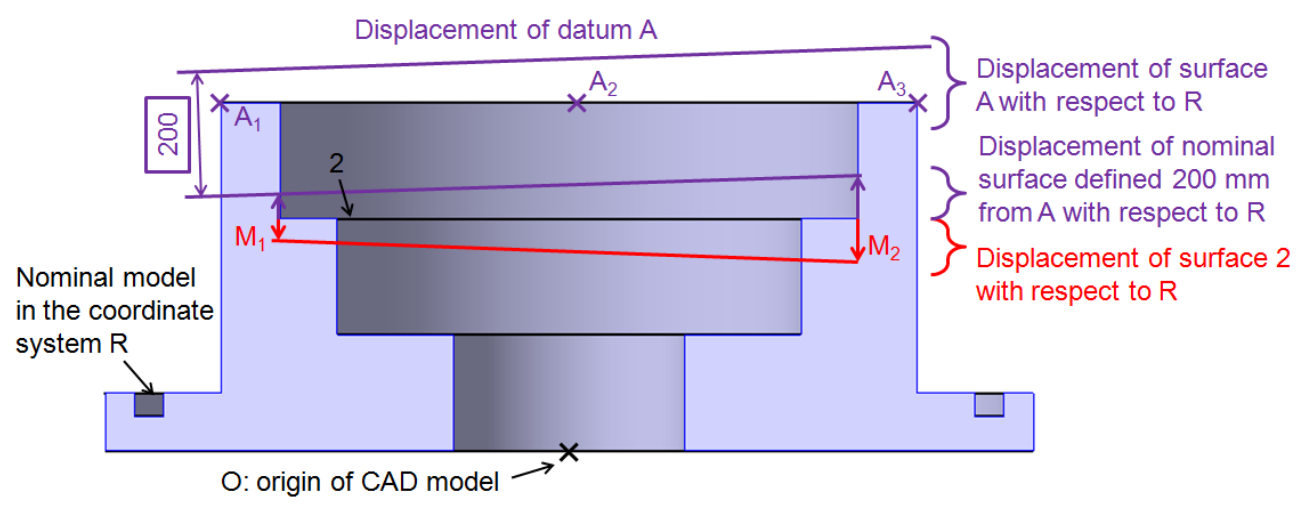

Figure 11: Decomposition of the functional requirement into a sum of displacements

Thus, one must study the distance of points $M_{i}$ between real surface 2 and nominal surface 2 defined $200 \mathrm{~mm}$ from $\mathrm{A}$. The maximum distance is calculated along the two directions $\overrightarrow{n_{i}}$ and $-\overrightarrow{n_{i}}, \overrightarrow{n_{i}}$ being the outside material normal to surface 2 at $M_{i} . M_{i}$ are the analysis points. The set $\left(M_{i}, \overrightarrow{n_{i}}\right)$ determines an analysis line.

These displacements must meet the functional requirement:

$$
\left|d_{2}\left(M_{i}, \vec{n}_{i}\right)_{/ A}\right| \leq \frac{T}{2} ;\left|d_{2}\left(M_{i},-\vec{n}_{i}\right)_{/ A}\right| \leq \frac{T}{2}
$$

where $\mathrm{T}$ is the tolerance which is prescribed by the functional requirement. $d_{2}\left(M_{i}, \overrightarrow{n_{i}}\right)_{/ A}$ stands for the maximum displacement of surface 2 at point $M_{i}$ in direction $\overrightarrow{n_{i}}$ with respect to the nominal surface, defined in relation to datum A. In this paper, only the displacement along $+\overrightarrow{n_{i}}$ will be calculated because the deviations are symmetrical. The points $M_{i}$ are obtained by discretizing the external boundary of surface 2 (Figure 10a).

$$
M_{1}\left(\begin{array}{c}
500 \\
0 \\
400
\end{array}\right) ; M_{2}\left(\begin{array}{c}
0 \\
500 \\
400
\end{array}\right) ; M_{3}\left(\begin{array}{c}
-500 \\
0 \\
+400
\end{array}\right) ; M_{4}\left(\begin{array}{c}
0 \\
-500 \\
+400
\end{array}\right)
$$

\subsubsection{Decomposition of the requirement}

The nominal model of the part is defined on $\mathrm{R}$.

Through the various machining operations, surface A is manufactured with a deviation with respect to $R$. Surface 2 is also manufactured with a deviation with respect to $R$.

Thus, in order to meet condition (4), one must determine, on the one hand, the displacement 
$d_{2}\left(M_{i}, \overrightarrow{n_{i}}\right)_{/ R}$ of the points $M_{i}$ which belong to the toleranced surface (surface 2 ) with respect to system $\mathrm{R}$ and, on the other hand, the displacement $d_{A}\left(M_{i},-\overrightarrow{n_{i}}\right)_{/ R}$ of the points $M_{i}$ which belong to nominal surface 2, which is defined $200 \mathrm{~mm}$ from A (Figure 11).

$$
d_{2}\left(M_{i}, \overrightarrow{n_{i}}\right)_{/ A}=d_{2}\left(M_{i}, \overrightarrow{n_{i}}\right)_{/ R}+d_{A}\left(M_{i},-\overrightarrow{n_{i}}\right)_{/ R}
$$

In the analysis line method, the datum system of the requirement must be represented as an isostatic datum system with a maximum of 6 points denoted $A_{j}$. Since A is a primary plane, 3 points are necessary and sufficient. In Section 4.5, we will show that the choice of these points is arbitrary.

Figure 10b illustrates these discretizations.

$$
A_{1}\left(\begin{array}{c}
0 \\
600 \\
600
\end{array}\right) ; A_{2}\left(\begin{array}{c}
600 \cos \left(30^{\circ}\right) \\
-300 \\
+600
\end{array}\right) ; A_{3}\left(\begin{array}{c}
-600 \cos \left(30^{\circ}\right) \\
-300 \\
+600
\end{array}\right)
$$

\subsubsection{Displacement of the nominal surface of the requirement}

The datum system of the requirement, denoted A, is created on the real surfaces of the part. Its shift with respect to the nominal $\mathrm{R}$ can be modeled as a displacement in $\mathrm{O} d \vec{O}=\left(\begin{array}{lll}u & v & w\end{array}\right)^{T}$ and a rotation $\vec{\Omega}=\left(\begin{array}{lll}\alpha & \beta & \gamma\end{array}\right)^{T}$. This deviation is due to the displacements of the 6 points $A_{j}$. The distance of each point $A_{j}$ to the nominal $\mathrm{R}$ is $d_{A}\left(A_{j}, \overrightarrow{n_{j}}\right)_{/ R}=d \overrightarrow{A_{j}} \cdot \overrightarrow{n_{j}}$, with:

$$
d \vec{A}_{j}=\overrightarrow{d O}+\vec{\Omega} \wedge \vec{A}_{j}
$$

$\overrightarrow{n_{j}}$ is the normal to nominal surface. The 6 components of the torsor $(u, v, w, \alpha, \beta$ and $\gamma)$ can be calculated from the 6 deviations of points $A_{j}$.

$$
\begin{aligned}
& d_{A}\left(A_{j}, \vec{n}_{j}\right)_{/ R}= \\
& \left(\begin{array}{llllll}
n_{x}, & n_{y}, & n_{z}, & -z n_{y}+y n_{z}, & -x n_{z}+z n_{x}, & -y n_{x}+x n_{y}
\end{array}\right) \\
& \left(\begin{array}{llllll}
u & v & w & \alpha & \beta & \gamma
\end{array}\right)^{T}
\end{aligned}
$$

If the system is isostatic, the matrix $\mathbf{T}_{\mathbf{A}}$ formed by these 6 equations can be inverted:

$$
\left(\begin{array}{llllll}
u & v & w & \alpha & \beta & \gamma
\end{array}\right)^{T}=\mathbf{T}_{\mathbf{A}}{ }^{-1}\left(\begin{array}{c}
d_{A}\left(A_{1}, \overrightarrow{n_{1}}\right) / R \\
\ldots \\
d_{A}\left(A_{6}, \overrightarrow{n_{6}}\right) / R
\end{array}\right)
$$

Now, one can express the displacement of the points $M_{i}$ which belong to the nominal surface defined in the datum system of requirement $A$ with respect to $R$.

$$
d \vec{M}_{i}=\overrightarrow{d O}+\vec{\Omega} \wedge O \vec{M}_{i}
$$

The displacement of the points $M_{i}$ belonging to the nominal is:

$$
d_{A}\left(M_{i},-\vec{n}_{i}\right)_{/ R}=d \vec{M}_{i} \cdot-\overrightarrow{n_{i}}=\left(\overrightarrow{d O}+\vec{\Omega} \wedge O \vec{M}_{i}\right) \cdot-\overrightarrow{n_{i}}
$$

This relation can be written in the linear form:

$$
d_{A}\left(M_{i},-\overrightarrow{n_{i}}\right)_{/ R}=\mathbf{T}_{\mathbf{M i}}\left(\begin{array}{llllll}
u & v & w & \alpha & \beta & \gamma
\end{array}\right)^{T}
$$

where $\mathbf{T}_{\mathbf{M i}}$ is the matrix defined for a point $M_{i}$ and a normal $\overrightarrow{n_{i}}$. The association of (8) and (11) leads to: 


$$
d_{A}\left(M_{i},-\overrightarrow{n_{i}}\right)_{/ R}=\sum_{j=1}^{p} k_{i j} d_{A}\left(A_{j}, \overrightarrow{n_{j}}\right)_{/ R}
$$

\subsubsection{The general transfer relation}

Once the requirement has been decomposed, the transfer relation is obtained by injecting (12) into (5):

$$
d_{2}\left(M_{i}, \overrightarrow{n_{i}}\right)_{/ A}=d_{2}\left(M_{i}, \overrightarrow{n_{i}}\right)_{/ R}+\sum_{j=1}^{p} k_{i j} d_{F}\left(A_{j}, \overrightarrow{n_{j}}\right)_{/ R}
$$

for each point $M_{i}$ and in each direction $n_{i}$.

The numerical application of the decomposition of the functional requirement into a weighted sum of point displacements is given in the annex (Section 6.1). The result is relation (34).

\subsection{Completion of the transfer}

\subsubsection{Principle of the transfer}

Each point $M_{i}$ or $A_{j}$ belongs to a surface which is manufactured in a phase. The transfer is performed sequentially from phase to phase, beginning with the last manufactured surface among all the surfaces included in the transfer relation.

\subsubsection{Transfer with a measurement operation}

In the transfer relation (34), the last manufactured surface is surface 2, which is manufactured in phase 30 in local WCS $R_{30.2}$ as described in Figure 3. The term to be decomposed in phase 30 is:

$$
d_{2}\left(M_{i}, \vec{z}\right)_{/ R}=d_{2}\left(M_{i}, \vec{z}\right)_{/ R_{30.2}}+d_{R_{30.2}}\left(M_{i}, \vec{z}\right)_{/ R}
$$

$d_{2}\left(M_{i}, \vec{z}\right) / R_{30.2}$ is the displacement of manufactured surface 2 with respect to the WCS of phase 30. This displacement is to be controlled through production specifications in that phase 30. $d_{R_{30.2}}\left(M_{i}, \vec{z}\right)_{/ R}$ is the displacement of point $M_{i}$ which is induced by the shifted position of WCS $R_{30.2}$ with respect to $\mathrm{R}$. This term requires the calculation of the displacement of WCS $R_{30.2}$ at point $M_{i}$ in direction $n_{i}$ with respect to $\mathrm{R}$.

That WCS $R_{30.2}$ is parallel to the primary plane which goes through $P_{30.1}, P_{30.2}$ and $P_{30.3}$. It is defined using secondary points $S_{30.1}$ and $S_{30.2}$, tertiary point $T_{30.1}$ and point $Q_{30.1}$. The deviation of WCS $R_{30.2}$ with respect to $\mathrm{R}$ is represented by vector

$\tau_{\mathbf{3 0 0}}=\left(\begin{array}{lllllll}u & v & w & \alpha & \beta & \gamma & \delta\end{array}\right)^{T}$. Vector $\tau_{\mathbf{3 0 0}}$ contains the components of the Small-Displacement Torsor of WCS $R_{30.2}$ with respect to $\mathrm{R}$ at point $\mathrm{O}$, as well as $\delta$, the difference between the actual distance from $P_{30}$ to $Q_{30.1}$ and the corresponding Theoretically Exact Dimension (TED). The deviation torsor of $R_{30.2}$ depends on the manufacturing dispersions of the isostatism points due to the previous phases: 


$$
\begin{aligned}
d_{R_{30.2}}\left(P_{30.1},-\vec{z}\right)_{/ R} & =\left(\overrightarrow{d O}+\vec{\Omega} \wedge O \vec{P}_{30.1}\right) \cdot \overrightarrow{-z}-\delta \\
d_{R_{30.2}}\left(P_{30.2},-\vec{z}\right)_{/ R} & =\left(\overrightarrow{d O}+\vec{\Omega} \wedge O{\overrightarrow{P_{30.2}}}\right) \cdot \overrightarrow{-z}-\delta \\
d_{R_{30.2}}\left(P_{30.3},-\vec{z}\right)_{/ R} & =\left(\overrightarrow{d O}+\vec{\Omega} \wedge O{\overrightarrow{P_{30.3}}}\right) \cdot \overrightarrow{-z}-\delta \\
d_{R_{30.2}}\left(S_{30.1}, \vec{y}\right)_{/ R} & =\left(\overrightarrow{d O}+\vec{\Omega} \wedge O \vec{S}_{30.1}\right) \cdot \vec{y} \\
d_{R_{30.2}}\left(S_{30.2}, \vec{x}\right)_{/ R} & =\left(\overrightarrow{d O}+\vec{\Omega} \wedge O \overrightarrow{S_{30.2}}\right) \cdot \vec{x} \\
d_{R_{30.2}}\left(T_{30.1}, \vec{x}\right)_{/ R} & =\left(\overrightarrow{d O}+\vec{\Omega} \wedge O{\overrightarrow{T_{30.1}}}\right) \cdot \vec{x} \\
d_{R_{30.2}}\left(Q_{30.1}, \vec{z}\right)_{/ R} & =\left(\overrightarrow{d O}+\vec{\Omega} \wedge O \overrightarrow{Q_{30.1}}\right) \cdot \vec{z}
\end{aligned}
$$

This system of equations enables one to calculate the 7 components of vector $\tau_{\mathbf{3 0 0}}$ as functions of the displacements of the 7 points. Let $\mathbf{T}_{\mathbf{3 0}}$ denote the isostatism influence matrix in phase 30, such that:

$$
\left(\begin{array}{c}
d_{R_{30.2}}\left(P_{30.1},-\vec{z}\right)_{/ R} \\
d_{R_{30.2}}\left(P_{30.2},-\vec{z}\right)_{/ R} \\
d_{R_{30.2}}\left(P_{30.3},-\vec{z}\right)_{/ R} \\
d_{R_{30.2}}\left(S_{30.1}, \vec{y}\right)_{/ R} \\
d_{R_{30.2}}\left(S_{30.2}, \vec{x}\right)_{/ R} \\
d_{R_{30.2}}\left(T_{30.1}, \vec{x}\right)_{/ R} \\
d_{R_{30.2}}\left(Q_{30.1}, \vec{z}\right)_{/ R}
\end{array}\right)=\mathbf{T}_{\mathbf{3 0}} \tau_{\mathbf{3 0 O}}
$$

The numerical application yields:

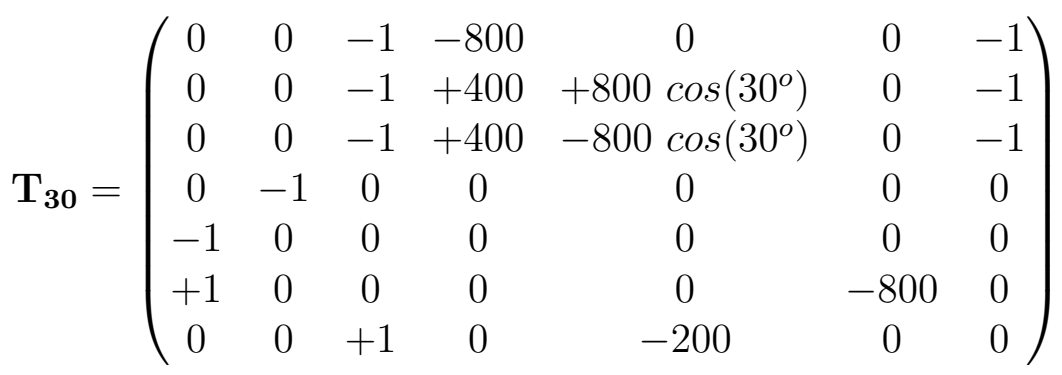

The displacement of the points $M_{i}$ of nominal surface 2 which is defined in this WCS $R_{30.2}$ is also of the form:

$$
d_{R_{30.2}}\left(M_{i}, \vec{n}_{i}\right)_{/ R}=\left(\overrightarrow{d O}+\vec{\Omega} \wedge O \vec{M}_{i}\right) \cdot \vec{z}
$$

Thus, this displacement can be expressed as a function of the 7 points:

$$
d_{R_{30.2}}\left(M_{i}, \vec{n}_{i}\right)_{/ R}=\mathbf{T}_{\mathbf{M}} \mathbf{T}_{\mathbf{3 0}}{ }^{-1}\left(\begin{array}{c}
d_{R_{30.2}}\left(P_{30.1},-\vec{z}\right)_{/ R} \\
d_{R_{30.2}}\left(P_{30.2},-\vec{z}\right)_{/ R} \\
\ldots \\
d_{R_{30.2}}\left(T_{30.1}, \vec{x}\right)_{/ R} \\
d_{R_{30.2}}\left(Q_{30.1}, \vec{z}\right)_{/ R}
\end{array}\right)
$$

For example, the displacement of the point $M_{3}$ is: 


$$
\begin{aligned}
& d_{R_{30.2}}\left(M_{3}, \vec{z}\right)_{/ R} \\
= & \left(\begin{array}{c}
0 \\
0.505 \\
-0.505 \\
0 \\
0 \\
0 \\
1
\end{array}\right) \times\left(\begin{array}{c}
d_{R_{30.2}}\left(P_{30.1},-\vec{z}\right)_{/ R} \\
d_{R_{30.2}}\left(P_{30.2},-\vec{z}\right)_{/ R} \\
d_{R_{30.2}}\left(P_{30.3},-\vec{z}\right)_{/ R} \\
d_{R_{30.2}}\left(S_{30.1}, \vec{y}\right)_{/ R} \\
d_{R_{30.2}}\left(S_{30.2}, \vec{x}\right)_{/ R} \\
d_{R_{30.2}}\left(T_{30.1}, \vec{x}\right)_{/ R} \\
d_{R_{30.2}}\left(Q_{30.1}, \vec{z}\right)_{/ R}
\end{array}\right)
\end{aligned}
$$

The transfer relation for the point $M_{3}$ becomes:

$$
\begin{aligned}
& d_{2}\left(M_{3}, \vec{z}\right)_{/ A}=0.505 d_{6}\left(P_{30.2},-\vec{z}\right)_{/ R} \\
& -0.505 d_{6}\left(P_{30.3},-\vec{z}\right)_{/ R}+d_{3}\left(Q_{30.1}, \vec{z}\right)_{/ R} \\
& -0.333 d_{1}\left(A_{1}, \vec{z}\right)_{/ R}+0.148 d_{1}\left(A_{2}, \vec{z}\right)_{/ R} \\
& -0.814 d_{1}\left(A_{3}, \vec{z}\right)_{/ R}+d_{2}\left(M_{3}, \vec{z}\right)_{/ R_{30.2}}
\end{aligned}
$$

\subsubsection{Continuation of the transfer by iteration}

This new transfer relation contains surfaces 1,3 and 6 , which are manufactured in phases 10, 20 and 10 respectively. The term to be decomposed in phase 20 is $d_{3}\left(Q_{30.1}, \vec{z}\right) / R$.

In the general case, without a measurement operation, the approach is the same, except that point $Q_{30.1}$ and distance $\delta$ do not exist. Therefore, there are 6 components of vector $\tau_{\mathbf{3 0 0}}$ to be determined from the displacements of the 6 points. The transfer is detailed in the annex (Section 6.3).

\subsection{Grouping of the points in the transfer relation}

The transfer relation can include a group $\mathrm{G}$ of 2 displacements of 2 points $P_{1}$ and $P_{2}$ belonging to the same plane $\mathrm{S}$ with normal $\vec{n}$.

$$
G=k_{1} d_{S}\left(P_{1}, \vec{n}\right)+k_{2} d_{S}\left(P_{2}, \vec{n}\right)
$$

In this case, the transfer relation can be simplified by expressing the displacements using the Small-Displacement Torsor of the surface:

$$
\begin{aligned}
G & =k_{1} d_{S}\left(P_{1}, \vec{n}\right)+k_{2} d_{S}\left(P_{2}, \vec{n}\right) \\
& =k_{1}(\overrightarrow{d O}+\vec{\Omega} \wedge \overrightarrow{O P}) \cdot \vec{n}+k_{2}\left(\overrightarrow{d O}+\vec{\Omega} \wedge O \vec{P}_{2}\right) \cdot \vec{n} \\
& =\left(k_{1}+k_{2}\right) \overrightarrow{d O} \cdot \vec{n}+\left(\vec{\Omega} \wedge\left(k_{1} O \vec{P}_{1}+k_{2} O \vec{P}_{2}\right)\right) \cdot \vec{n} \\
& =\left(k_{1}+k_{2}\right)\left(\overrightarrow{d O}+\vec{\Omega} \wedge \frac{k_{1} O \vec{P}_{1}+k_{2} O \vec{P}_{2}}{k_{1}+k_{2}}\right) \cdot \vec{n} \\
& =\left(k_{1}+k_{2}\right) d \vec{P}_{e q} \cdot \vec{n}
\end{aligned}
$$

where $P_{e q}$ defined by $O \vec{P}_{e q}=\frac{k_{1} O \overrightarrow{P P}_{1}+k_{2} O \vec{P}_{2}}{k_{1}+k_{2}}$ is the barycenter of $P_{1}$ and $P_{2}$ weighted by coefficients $k_{1}$ and $k_{2}$. The relation can be simplified by defining a new equivalent analysis point.

$$
\begin{aligned}
G & =k_{1} d_{S}\left(P_{1}, \vec{n}\right)+k_{2} d_{S}\left(P_{2}, \vec{n}\right) \\
& =\left(k_{1}+k_{2}\right) d_{S}\left(P_{e q}, \vec{n}\right)
\end{aligned}
$$


The numerical application given in the annex (Section 6.3) is an example of such point groupings. In the case $k_{1}+k_{2}=0$, this simplification is not possible because the equivalent point would be at infinity. Nevertheless, expressing the displacements using the surface's SmallDisplacement Torsor, the relation simplifies to:

$$
\begin{aligned}
G & =k\left(d_{S}\left(P_{1}, \vec{n}\right)-d_{S}\left(P_{2}, \vec{n}\right)\right) \\
& =k\left(\left(\overrightarrow{d O}+\vec{\Omega} \wedge \overrightarrow{O P_{1}}\right) \cdot \vec{n}-\left(\overrightarrow{d O}+\vec{\Omega} \wedge \overrightarrow{O P}_{2}\right) \cdot \vec{n}\right) \\
& =k\left(\overrightarrow{d O} \cdot \vec{n}-\overrightarrow{d O} \cdot \vec{n}+\left(\vec{\Omega} \wedge\left(\overrightarrow{O P_{1}}-O \vec{P}_{2}\right)\right) \cdot \vec{n}\right) \\
& =k\left(\vec{\Omega} \wedge \vec{P}_{1} P_{2}\right) \cdot \vec{n}=\vec{\Omega} \cdot\left(k \vec{P}_{1} \wedge \vec{n}\right)=\vec{\Omega} \cdot \vec{\rho}
\end{aligned}
$$

The translation of the surface (modeled by vector $\overrightarrow{d O}$ ) does not affect the result, which is influenced only by the rotation of the surface arround $\vec{\rho}=k \overrightarrow{P_{1} P_{2}} \wedge \vec{n}$.

\subsection{Production specification synthesis}

\subsubsection{Principle}

At the end of the transfer and after point grouping, the relation is left with only displacements of surfaces with respect to their respective manufacturing datum systems. For example, the relation obtained in (45) for point $M_{3}$ is:

$$
\begin{aligned}
& d_{2}\left(M_{3}, \vec{z}\right)_{/ A}=0.505 d_{6}\left(P_{30 \text { equ. } 3},-\vec{z}\right) / R_{10} \\
& -0.505 d_{6}\left(P_{30.3},-\vec{z}\right)_{/ R_{10}}+d_{1}\left(P_{20 \text { equ }}, \vec{z}\right)_{/ R_{10}} \\
& -d_{1}\left(A_{\text {equ. } 3}, \vec{z}\right)_{/ R_{10}}+d_{3}\left(Q_{30.1}, \vec{z}\right)_{/ R_{20}} \\
& +d_{2}\left(M_{3}, \vec{z}\right)_{/ R_{30.2}}
\end{aligned}
$$

One must still determine the production specifications which allow the values of the displacements of each surface to be controlled and to write the condition as a function of the tolerances.

\subsubsection{Term of the form $d_{S}(P, \vec{n}) / R_{N}$}

One must quantify the maximum displacement of point $\mathrm{P}$ belonging to surface $\mathrm{S}$ manufactured in phase $\mathrm{N}$.

If point $\mathrm{P}$ lies within the limits of surface $\mathrm{S}$, a position specification must be applied to surface $\mathrm{S}$ with respect to the datum system of phase $\mathrm{N}$. This position tolerance is denoted $t_{p o s, S}$.

The maximum displacement due to manufacturing defects is:

$$
d_{S}(P, \vec{n})_{/ R_{N}}=\frac{1}{2} t_{p o s, S}
$$

If point $\mathrm{P}$ is outside the limits of surface $\mathrm{S}$, both a position specification $t_{p o s, S}$ and an orientation specification $t_{\text {ori }, S}$ must be applied to surface $S$ with respect to the datum system of phase $\mathrm{N}$. The maximum displacement is:

$$
d_{S}(P, \vec{n})_{/ R_{N}}=\frac{1}{2} t_{p o s, S}+\frac{L}{E} t_{o r i, S}
$$

where $\mathrm{L}$ is the lever arm between point $\mathrm{P}$ and surface $\mathrm{S}$ and $\mathrm{E}$ is the extent of surface $\mathrm{S}$ in the direction of this lever arm. 


\subsubsection{Terms of the form $d_{S}\left(P_{i}, \vec{n}\right)_{/ R_{N}}-d_{S}\left(P_{j}, \vec{n}\right)_{/ R_{N}}$}

If the transfer relation involves terms of the form

$d_{S}\left(P_{i}, \vec{n}\right)_{/ R_{N}}-d_{S}\left(P_{j}, \vec{n}\right)_{/ R_{N}}$, one must apply an orientation specification of surface $\mathrm{S}$ with respect to the datum system of phase $\mathrm{N}$. This orientation tolerance is denoted $t_{\text {ori }, S}$. The worst-case influence of this angle is:

$$
d_{S}\left(P_{i}, \vec{n}\right)_{/ R_{N}}-d_{S}\left(P_{j}, \vec{n}\right)_{/ R_{N}}=\frac{L}{E} t_{\text {ori }, S}
$$

where $\mathrm{L}$ is the length $P_{i} P_{j}$ and $\mathrm{E}$ is the extent of surface $\mathrm{S}$ in the direction given by $\left(P_{i} P_{j}\right)$.

\subsection{Final transfer relation}

The transfer of the functional requirement is fully described in the annex (Section 6). The worst-case condition obtained is relation (48) given here again:

$$
t_{p o s, 3}+t_{\text {pos }, 2}+0.876 t_{\text {ori }, 6}+1.166 t_{\text {ori }, 1} \leq T
$$

One must still choose manufacturing tolerances such that condition (30) will be met. A similar relation is determined for each functional requirement, and this set of inequations enables the values of the tolerances to be optimized for a chosen objective function.

The specifications obtained are mentioned on the phase drawings (Figure 12). 


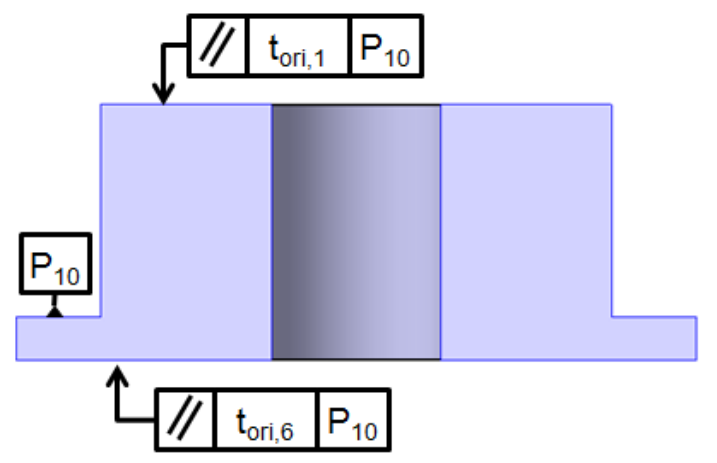

(a) Phase 10

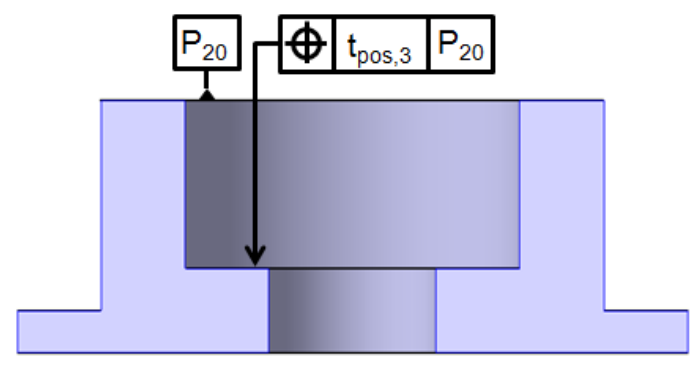

(b) Phase 20

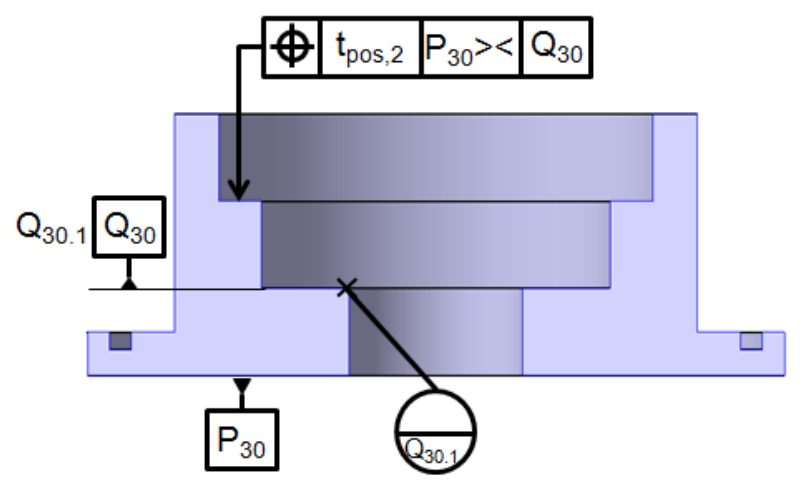

(c) Phase 30

Figure 12: Specification synthesis 


\section{Conclusions}

The proposed transfer method enables orientation effects to be taken into account in manufacturing transfer.

The specificity of machining in a WCS defined through probing has been included.

An explicit three-dimensional linear relation has been determined. This relation, which is of the form

$\Sigma k_{i} t_{i} \leq \frac{T}{2}$, gives the dependence between the displacement of the toleranced surface and the manufacturing tolerances. It shows the advantage of a 3D transfer compared to a $1 \mathrm{D}$ transfer. Indeed, the calculation showed that two orientation tolerances $t_{\text {ori }, 1}$ and $t_{\text {ori }, 6}$ must be controlled in phase 10. Besides, the sensitivity of these orientation tolerances in the transfer is of the same order of magnitude as the sensitivity of the position defects. Therefore, neglecting these effects without qualifying the process would have been an error.

The methodology is applied here for NC turning. It is also applicable for any machining operations, as long as setting-up is made of isostatic points, or can be modeled by isostatic points. However, the developments presented in this paper cannot be envisaged in an industrial context without a calculation tool.

This is the reason why a new methodology, based on the Computer Aided Tolerancing (CAT) software ANATOLE of Airbus Group Innovations, is currently being developed. This will provide manufacturing engineers with the necessary calculation tools to determine the production specifications. The case of a local WCS defined by probing is included. The process plan presented in this paper was addressed using this method. The results proposed by the software are identical to those which are given by the method described here. In the future, this methodology will be proposed to manufacturing engineers.

\section{Annex: the complete manufacturing transfer}

\subsection{Decomposition of the requirement}

The objective is to determine the displacement of the points $M_{i}$ belonging to the nominal surface defined in the datum system of the requirement with respect to datum $\mathrm{R}$.

The relations derived from (7) which characterize the differences between the datum system of the requirement and the nominal model in $\mathrm{R}$ at points $A_{j}$ can be grouped in the form of a matrix. Datum plane A limits only components $w, \alpha$ and $\beta$ of the Small-Displacement Torsor, leading to:

$$
\begin{aligned}
& \left(\begin{array}{l}
d_{1}\left(A_{1}, \vec{z}\right)_{/ R} \\
d_{1}\left(A_{2}, \vec{z}\right)_{/ R} \\
d_{1}\left(A_{3}, \vec{z}\right)_{/ R}
\end{array}\right)=\mathbf{T}_{\mathbf{F}}\left(\begin{array}{lll}
w & \alpha & \beta
\end{array}\right)^{T}
\end{aligned}
$$

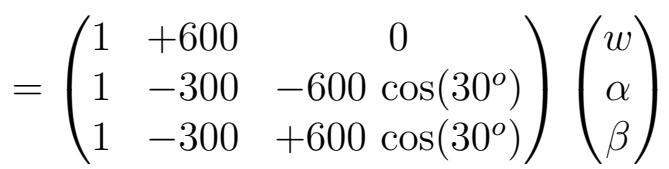

Similarly, the deviation at point $M_{3}$ located $200 \mathrm{~mm}$ from A is characterized by matrix $\mathrm{T}_{\mathrm{M}_{3}}$.

$$
\mathbf{T}_{\mathbf{M}_{3}}=\left(\begin{array}{llllll}
0 & 0 & -1 & 0 & -500 & 0
\end{array}\right)
$$

The displacement of the point $M_{3}$ belonging to the nominal surface with respect to $\mathrm{R}$ is determined from (31) and (32): 


$$
\begin{aligned}
& d_{A}\left(M_{3},-\vec{z}\right)_{/ R}=\mathbf{T}_{\mathbf{M}_{3}}\left(\begin{array}{lll}
w & \alpha & \beta
\end{array}\right)^{T} \\
& =\mathbf{T}_{\mathbf{M}_{3}} \mathbf{T}_{\mathbf{F}}{ }^{-1}\left(\begin{array}{l}
d_{1}\left(A_{1}, \vec{z}\right)_{/ R} \\
d_{1}\left(A_{2}, \vec{z}\right)_{/ R} \\
d_{1}\left(A_{3}, \vec{z}\right)_{/ R}
\end{array}\right)=-0.333 d_{1}\left(A_{1}, \vec{z}\right)_{/ R} \\
& +0.148 d_{1}\left(A_{2}, \vec{z}\right)_{/ R}-0.814 d_{1}\left(A_{3}, \vec{z}\right)_{/ R}
\end{aligned}
$$

Relation (33) is inserted into relation (5). The transfer relation for the point $M_{3}$ becomes:

$$
\begin{aligned}
& d_{2}\left(M_{3}, \vec{z}\right)_{/ A}=d_{2}\left(M_{3}, \vec{z}\right)_{/ R}-0.333 d_{1}\left(A_{1}, \vec{z}\right)_{/ R} \\
& +0.148 d_{1}\left(A_{2}, \vec{z}\right)_{/ R}-0.814 d_{1}\left(A_{3}, \vec{z}\right)_{/ R}
\end{aligned}
$$

\subsection{Transfer in phase 30}

This relation involves points belonging to surfaces 1 and 2 , which are manufactured in phases 10 and 30 respectively. The transfer begins with phase 30, which is manufactured last (this aspect is developed in 4.4.2.)

\subsection{Transfer in phase 20}

In the transfer relation following the study of phase 30 , it is surface 3 which is manufactured last in phase $20 . d_{3}\left(Q_{30.1}, \vec{z}\right)_{/ R}$ is decomposed as follows:

$$
d_{3}\left(Q_{30.1}, \vec{z}\right)_{/ R}=d_{3}\left(Q_{30.1}, \vec{z}\right)_{/ R_{20}}+d_{R_{20}}\left(Q_{30.1}, \vec{z}\right)_{/ R}
$$

$d_{3}\left(Q_{30.1}, \vec{z}\right) / R_{20}$ is the displacement of manufactured surface 3 with respect to the datum of phase 20. This displacement is to be controlled using production specifications in phase 20 . $d_{R_{20}}\left(Q_{30.1}, \vec{z}\right)_{/ R}$ is the displacement of point $Q_{30.1}$ induced by the displacement of the isostatism points of phase 20 with respect to $\mathrm{R}$.

The coordinates of points $P_{20.1}, P_{20.2}, P_{20.3}, S_{20.1}, S_{20.2}$ and $T_{20.1}$ are grouped in the influence matrix $\mathbf{T}_{\mathbf{2 0}}$ using relation (7):

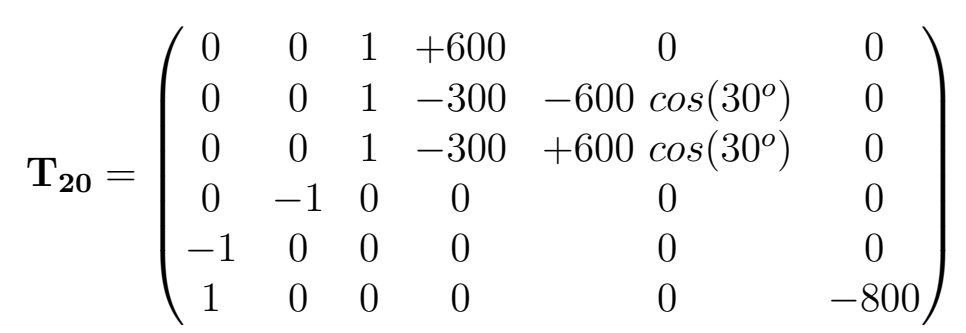

$\mathbf{T}_{\mathbf{2 0}}$ leads to the expression of the displacement of point $Q_{30.1}$ induced by the displacements of the isostatism points of phase 20 with respect to $R$ : 


$$
\begin{aligned}
& d_{R_{20}}\left(Q_{30.1}, \vec{z}\right)_{/ R}=\mathbf{T}_{\mathbf{Q}_{\mathbf{3 0 . 1}}} \mathbf{T}_{\mathbf{2 0}}{ }^{-1}\left(\begin{array}{l}
d\left(P_{20.1}\right) \\
d\left(P_{20.2}\right) \\
d\left(P_{20.3}\right) \\
d\left(S_{20.1}\right) \\
d\left(S_{20.2}\right) \\
d\left(T_{20.1}\right)
\end{array}\right) \\
& =\left(\begin{array}{llllll}
0 & 0 & 1 & 0 & -200 & 0
\end{array}\right) \mathbf{T}_{\mathbf{2 0}}{ }^{-1}\left(\begin{array}{l}
d\left(P_{20.1}\right) \\
d\left(P_{20.2}\right) \\
d\left(P_{20.3}\right) \\
d\left(S_{20.1}\right) \\
d\left(S_{20.2}\right) \\
d\left(T_{20.1}\right)
\end{array}\right) \\
& =0.333 d_{1}\left(P_{20.1}, \vec{z}\right)_{/ R}+0.526 d_{1}\left(P_{20.2}, \vec{z}\right)_{/ R} \\
& +0.141 d_{1}\left(P_{20.3}, \vec{z}\right)_{/ R}
\end{aligned}
$$

Introducing (37) into (21), the transfer relation for the point $M_{3}$ becomes:

$$
\begin{aligned}
& d_{2}\left(M_{3}, \vec{z}\right)_{/ A}=0.505 d_{6}\left(P_{30.2},-\vec{z}\right)_{/ R} \\
& -0.505 d_{6}\left(P_{30.3},-\vec{z}\right)_{/ R}+0.333 d_{1}\left(P_{20.1}, \vec{z}\right)_{/ R} \\
& +0.526 d_{1}\left(P_{20.2}, \vec{z}\right)_{/ R}+0.141 d_{1}\left(P_{20.3}, \vec{z}\right)_{/ R} \\
& -0.333 d_{1}\left(A_{1}, \vec{z}\right)_{/ R}+0.148 d_{1}\left(A_{2}, \vec{z}\right)_{/ R} \\
& -0.814 d_{1}\left(A_{3}, \vec{z}\right)_{/ R}+d_{3}\left(Q_{30.1}, \vec{z}\right)_{/ R_{20}} \\
& +d_{2}\left(M_{3}, \vec{z}\right)_{/ R_{30.2}}
\end{aligned}
$$

Since points $A_{1}, A_{2}, A_{3}, P_{20.1}, P_{20.2}$ and $P_{20.3}$ all belong to surface 1, it is possible to group their influences by carrying out a barycenter calculation, as presented in 4.5. However, the sum of the coefficients of the displacements of these points is zero. Therefore, these points end up in groups of 3 .

The set-up points $P_{20.1}, P_{20.2}$ and $P_{20.3}$ in phase 20 can be grouped into an equivalent point $P_{20 e q u}$.

$$
\begin{aligned}
& d_{2}\left(M_{3}, \vec{z}\right)_{/ A}=\ldots+0.333 d_{1}\left(P_{20.1}, \vec{z}\right)_{/ R} \\
& +0.526 d_{1}\left(P_{20.2}, \vec{z}\right)_{/ R}+0.141 d_{1}\left(P_{20.3}, \vec{z}\right)_{/ R} \\
& =\ldots+1 d_{1}\left(P_{20 \text { equ }}, \vec{z}\right)_{/ R}
\end{aligned}
$$

The barycenter calculation leads to the coordinates of points $P_{20 e q u}$ located across from point $Q_{30.1}$ :

$$
P_{20 e q u}\left(\begin{array}{c}
200 \\
0 \\
600
\end{array}\right)
$$

For points $A_{1}, A_{2}$ and $A_{3}$, an equivalent point $A_{\text {equ.3 }}$ is determined :

$$
\begin{aligned}
& d_{2}\left(M_{3}, \vec{z}\right)_{/ A}=\ldots-0.333 d_{1}\left(A_{1}, \vec{z}\right)_{/ R} \\
& +0.148 d_{1}\left(A_{2}, \vec{z}\right)_{/ R}-0.814 d_{1}\left(A_{3}, \vec{z}\right)_{/ R} \\
& =\ldots-1 d_{1}\left(A_{\text {equ. } 3}, \vec{z}\right)_{/ R}
\end{aligned}
$$

The barycenter calculation leads to the coordinates of points $A_{\text {equ.3: }}$ : 


$$
A_{\text {equ. } 3}\left(\begin{array}{c}
-500 \\
0 \\
600
\end{array}\right)
$$

This point is located across from point $M_{3}$. The transfer relation for the point $M_{3}$ becomes:

$$
\begin{aligned}
& d_{2}\left(M_{3}, \vec{z}\right)_{/ A}=0.505 d_{6}\left(P_{30.2},-\vec{z}\right)_{/ R} \\
& -0.505 d_{6}\left(P_{30.3},-\vec{z}\right)_{/ R}+d_{1}\left(P_{20 e q u}, \vec{z}\right)_{/ R} \\
& -d_{1}\left(A_{\text {equ. } 3}, \vec{z}\right)_{/ R}+d_{3}\left(Q_{30.1}, \vec{z}\right)_{/ R_{20}} \\
& +d_{2}\left(M_{3}, \vec{z}\right)_{/ R_{30.2}}
\end{aligned}
$$

This new transfer relation contains surfaces 1 and 6 , which are manufactured in phase 10 . The terms to be decomposed in phase 10 are $d_{6}\left(P_{30.2},-\vec{z}\right)_{/ R}$,

$d_{6}\left(P_{30.3},-\vec{z}\right)_{/ R}, d_{1}\left(P_{20 e q u}, \vec{z}\right)_{/ R}$ and $d_{1}\left(A_{\text {equ. } 3}, \vec{z}\right)_{/ R}$.

\subsection{The study of phase 10}

$d_{6}\left(P_{30.2},-\vec{z}\right)_{/ R}, d_{6}\left(P_{30.3},-\vec{z}\right)_{/ R}, d_{1}\left(P_{20 \text { equ }}, \vec{z}\right)_{/ R}$ and $d_{1}\left(A_{\text {equ. } 3}, \vec{z}\right)_{/ R}$ are decomposed as follows:

$$
\begin{aligned}
d_{6}\left(P_{30.2},-\vec{z}\right)_{/ R} & =d_{6}\left(P_{30.2},-\vec{z}\right) / R_{10} \\
& +d_{R_{10}}\left(P_{30.2},-\vec{z}\right) / R \\
d_{6}\left(P_{30.3},-\vec{z}\right)_{/ R} & =d_{6}\left(P_{30.3},-\vec{z}\right) / R_{10} \\
& +d_{R_{10}}\left(P_{30.3},-\vec{z}\right)_{/ R} \\
d_{1}\left(P_{20 e q u}, \vec{z}\right)_{/ R} & =d_{1}\left(P_{20 \text { equ }}, \vec{z}\right)_{/ R_{10}} \\
& +d_{R_{10}}\left(P_{20 e q u}, \vec{z}\right) / R \\
d_{1}\left(A_{\text {equ. } 3}, \vec{z}\right)_{/ R} & =d_{1}\left(A_{\text {equ. }}, \vec{z}\right) / R_{10} \\
& +d_{R_{10}}\left(A_{\text {equ. } 3}, \vec{z}\right)_{/ R}
\end{aligned}
$$

$d_{6}\left(P_{30.2},-\vec{z}\right) / R_{10}, d_{6}\left(P_{30.3},-\vec{z}\right) / R_{10}$,

$d_{1}\left(P_{20 \text { equ }}, \vec{z}\right) / R_{10}$ and $d_{1}\left(A_{\text {equ. } 3}, \vec{z}\right) / R_{10}$ are the displacements of manufactured surfaces 1 and 6 with respect to the datum of phase 10 . These displacements are to be controlled using production specifications.

$d_{R_{10}}\left(P_{30.2},-\vec{z}\right)_{/ R}, d_{R_{10}}\left(P_{30.3},-\vec{z}\right)_{/ R}$,

$d_{R_{10}}\left(P_{20 \text { equ }}, \vec{z}\right)_{/ R}$ and $d_{R_{10}}\left(A_{\text {equ. } 3}, \vec{z}\right)_{/ R}$ are the displacements of points $P_{30.2}, P_{30.3}, P_{20 e q u}$ and $A_{\text {equ. } 3}$ induced by the displacements of the isostatism points of phase 10 with respect to $\mathrm{R}$.

The coordinates of points $P_{10.1}, P_{10.2}, P_{10.3}, S_{10.1}, S_{10.2}$ and $T_{10.1}$ are grouped in the influence matrix $\mathbf{T}_{\mathbf{1 0}}$ using relation (7):

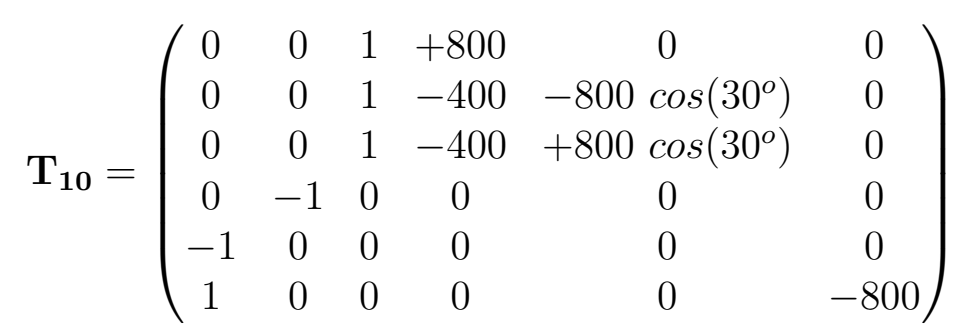

$\mathbf{T}_{\mathbf{1 0}}$ leads to the expression of the displacements of points $P_{30.2}, P_{30.3}, P_{20 \text { equ }}$ and $A_{\text {equ. } 3}$ induced by the displacements of the isostatism points of phase 10 with respect to R. For example, for point $P_{20 e q u}$ : 


$$
\begin{aligned}
& d_{R_{10}}\left(P_{20 \text { equ }}, \vec{z}\right)_{/ R}=\mathbf{T}_{\mathbf{P}_{\text {20equ }}} \mathbf{T}_{\mathbf{1 0}}{ }^{-1}
\end{aligned}
$$

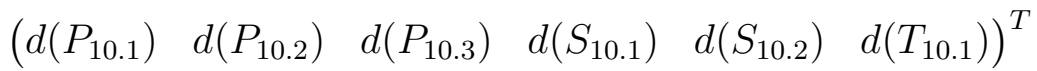

$$
\begin{aligned}
& =\left(\begin{array}{llllll}
0 & 0 & 1 & 0 & -200 & 0
\end{array}\right) \mathbf{T}_{\mathbf{1 0}}{ }^{-1}
\end{aligned}
$$

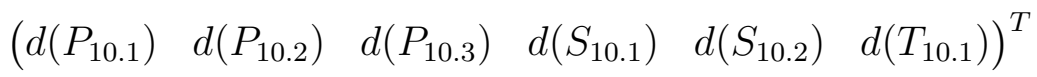

$$
\begin{aligned}
& =0
\end{aligned}
$$

Inserting (44) into (41), the transfer relation becomes:

$$
\begin{aligned}
& \left(\begin{array}{l}
d_{2}\left(M_{1}, \vec{z}\right) / A \\
d_{2}\left(M_{2}, \vec{z}\right)_{/ A} \\
d_{2}\left(M_{3}, \vec{z}\right)_{/ A} \\
d_{2}\left(M_{4}, \vec{z}\right)_{/ A}
\end{array}\right)=\left(\begin{array}{c}
-0.217 d_{6}\left(P_{30.2},-\vec{z}\right) / R_{10} \\
-0.064 d_{6}\left(P_{30 e q u .2},-\vec{z}\right) / R_{10} \\
+0.505 d_{6}\left(P_{30.2},-\vec{z}\right) / R_{10} \\
+0.353 d_{6}\left(P_{30 \text { equ.4 }},-\vec{z}\right) / R_{10}
\end{array}\right) \\
& +\left(\begin{array}{l}
+0.217 \\
+0.064 \\
-0.505 \\
-0.353
\end{array}\right) d_{6}\left(P_{30.3},-\vec{z}\right) / R_{10} \\
& +\left(\begin{array}{l}
1 \\
1 \\
1 \\
1
\end{array}\right) d_{1}\left(P_{20 \text { equ }}, \vec{z}\right) / R_{10}+\left(\begin{array}{l}
-d_{1}\left(A_{\text {equ. }}, \vec{z}\right) / R_{10} \\
-d_{1}\left(A_{\text {equ. }}, \vec{z}\right) / R_{10} \\
-d_{1}\left(A_{\text {equ.3 }}, \vec{z}\right) / R_{10} \\
-d_{1}\left(A_{\text {equ.4 }}, \vec{z}\right) / R_{10}
\end{array}\right) \\
& +\left(\begin{array}{l}
1 \\
1 \\
1 \\
d_{2}\left(M_{1}, \vec{z}\right) / R_{30.2} \\
d_{2}\left(M_{2}, \vec{z}\right) / R_{30.2} \\
d_{2}\left(M_{3}, \vec{z}\right) / R_{30.2} \\
d_{2}\left(M_{4}, \vec{z}\right) / R_{30.2}
\end{array}\right)
\end{aligned}
$$

All the remaining displacements in the relation are expressed in the datum systems of the different phases. This completes the transfer; only the specifications remain to be chosen.

\subsection{Specification synthesis}

Following the method proposed in Section 4.6.3, orientation specifications are prescribed on surfaces 1 and 6 , and the relation becomes:

$$
\begin{aligned}
& \left(\begin{array}{l}
d_{2}\left(M_{1}, \vec{z}\right)_{/ A} \\
d_{2}\left(M_{2}, \vec{z}\right)_{/ A} \\
d_{2}\left(M_{3}, \vec{z}\right)_{/ A} \\
d_{2}\left(M_{4}, \vec{z}\right)_{/ A}
\end{array}\right)=\left(\begin{array}{l}
1 \\
1 \\
1 \\
1
\end{array}\right) d_{3}\left(Q_{30.1}, \vec{z}\right)_{/ R_{20}} \\
& +\left(\begin{array}{l}
d_{2}\left(M_{1}, \vec{z}\right)_{/ R_{30.2}} \\
d_{2}\left(M_{2}, \vec{z}\right)_{/ R_{30.2}} \\
d_{2}\left(M_{3}, \vec{z}\right) / R_{30.2} \\
d_{2}\left(M_{4}, \vec{z}\right) / R_{30.2}
\end{array}\right)+\left(\begin{array}{l}
0.188 \\
0.337 \\
0.438 \\
0.337
\end{array}\right) t_{\text {ori }, 6} \\
& +\left(\begin{array}{l}
0.250 \\
0.449 \\
0.583 \\
0.449
\end{array}\right) t_{\text {ori }, 1}
\end{aligned}
$$

Since points $M_{i}$ are located within the limits of surface 2 and point $Q_{30.1}$ is located within the limits of surface 3, the method developed in Section 4.6.2 requires that position specifications $t_{p o s, 2}$ and $t_{p o s, 3}$ be prescribed on surfaces 2 and 3 , and the transfer relation becomes: 


$$
\begin{aligned}
& \left(\begin{array}{l}
d_{2}\left(M_{1}, \vec{z}\right)_{/ A} \\
d_{2}\left(M_{2}, \vec{z}\right)_{/ A} \\
d_{2}\left(M_{3}, \vec{z}\right)_{/ A} \\
d_{2}\left(M_{4}, \vec{z}\right)_{/ A}
\end{array}\right)=\left(\begin{array}{c}
0.5 \\
0.5 \\
0.5 \\
0.5
\end{array}\right) t_{\text {pos }, 3} \\
& +\left(\begin{array}{l}
0.5 \\
0.5 \\
0.5 \\
0.5
\end{array}\right) t_{\text {pos }, 2}+\left(\begin{array}{l}
0.188 \\
0.337 \\
0.438 \\
0.337
\end{array}\right) t_{\text {ori }, 6}+\left(\begin{array}{l}
0.250 \\
0.449 \\
0.583 \\
0.449
\end{array}\right) t_{\text {or } i, 1}
\end{aligned}
$$

The most severe condition is the relation concerning point $M_{3}$. Therefore, the sufficient condition to be satisfied is:

$$
t_{\text {pos }, 3}+t_{\text {pos }, 2}+0.876 t_{\text {ori }, 6}+1.166 t_{\text {ori }, 1} \leq T
$$

\section{Acknowledgements}

This work was supported by Snecma, company of Safran.

\section{References}

[1] Anselmetti, B.: CLIC: A method for geometrical specification of products. Geometric Tolerancing of Products pp. 207-239 (2010)

[2] Anselmetti, B.: Manuel de tolérancement (Volume 4) - Cotation de fabrication avec les normes ISO. Hermès Sciences (2010)

[3] Anselmetti, B.: Part optimization and tolerances synthesis. The International Journal of Advanced Manufacturing Technology 48(9-12), 1221-1237 (2010)

[4] Anselmetti, B.: ISO manufacturing tolerancing: three-dimensional transfer with analysis line method. The International Journal of Advanced Manufacturing Technology 61(9-12), 1085-1099 (2012)

[5] Ballot, E., Bourdet, P.: An integrated framework for 3D tolerance chains in design and manufacturing. Procedings of DETC'01 (2001). ASME 2001 Design Engineering Technical Conferences and Computers and Inbformation in Engineering Conference

[6] Bourdet, P.: Chaînes de cotes de fabrication. Journal L'ingénieur et le technicien de l'enseignement technique (191) (1973)

[7] Bourdet, P., Mathieu, L., Lartigue, C., Ballu, A.: The concept of the Small Displacement Torsor in metrology. Series on Advances in Mathematics for Applied Sciences 40, 110-122 (1996)

[8] Chase, K.W., Parkinson, A.R.: A survey of research in the application of tolerance analysis to the design of mechanical assemblies. Research in Engineering design 3(1), 23-37 (1991)

[9] Davidson, J., Mujezinovic, A., Shah, J.: A new mathematical model for geometric tolerances as applied to round faces. Journal of mechanical design 124(4), 609-622 (2002) 
[10] Haghighi, P., Mohan, P., Shah, J.J., Davidson, J.K.: A framework for explicating formal geometrical and dimensional tolerances schema from manufacturing process plans for three-dimensional conformance analysis. Journal of Computing and Information Science in Engineering 15(2), 021,003 (2015)

[11] ISO: 1101:2013 - Geometrical Product Specifications (GPS) - Geometrical tolerancing — Tolerances of form, orientation, location and run-out. International Standard

[12] ISO: 5459:2011 - Geometrical Product Specifications (GPS) - Geometrical tolerancing Datums and datum systems. International Standard

[13] Kusiak, A., Feng, C.X.: Deterministic tolerance synthesis: a comparative study. Computer-aided design 27(10), 759-768 (1995)

[14] Laifa, M., Sai, W.B., Hbaieb, M.: Evaluation of machining process by integrating 3D manufacturing dispersions, functional constraints, and the concept of small displacement torsors. The International Journal of Advanced Manufacturing Technology 71(5-8), 1327$1336(2014)$

[15] Salomons, O., Haalboom, F., Poerink, H.J., Van Slooten, F., Van Houten, F., Kals, H.: A computer aided tolerancing tool II: Tolerance analysis. Computers in Industry 31(2), $175-186$ (1996)

[16] Salomons, O., Poerink, H.J., Haalboom, F., Van Slooten, F., Van Houten, F., Kals, H.: A computer aided tolerancing tool I: Tolerance specification. Computers in Industry 31(2), 161-174 (1996)

[17] Salomons, O.W., Van Houten, F.J., Kals, H.J.: Current status of CAT systems. In: Geometric design tolerancing: Theories, standards and applications, pp. 438-452. Springer (1998)

[18] Vignat, F.: Contribution à l'élaboration d'un modèle 3D de simulation de fabrication pour l'analyse et la synthèse des tolérances, le modèle MMP. Ph.D. thesis, Grenoble, INPG (2005)

[19] Vignat, F., Villeneuve, F.: Simulation of the manufacturing process, generation of a Model of the Manufactured Parts. In: Digital Enterprise Technology, pp. 545-552. Springer (2007)

[20] Villeneuve, F., Vignat, F.: Simulation of the manufacturing process in a tolerancing point of view: Generic resolution of the positioning problem. In: Models for computer aided tolerancing in design and manufacturing, pp. 179-189. Springer (2007) 